\title{
Response Disengagement on a Spatial Self-Ordered Sequencing Task: Effects of Regionally Selective Excitotoxic Lesions and Serotonin Depletion within the Prefrontal Cortex
}

\author{
Susannah C. Walker, ${ }^{1,2}$ Trevor W. Robbins, ${ }^{1,2}$ and Angela C. Roberts ${ }^{2,3}$ \\ ${ }^{1}$ Department of Experimental Psychology and ${ }^{2}$ Behavioural and Clinical Neuroscience Institute, University of Cambridge, Cambridge CB2 3EB, United \\ Kingdom, and ${ }^{3}$ Department of Physiology, Development and Neuroscience, University of Cambridge, Cambridge CB2 3DY, United Kingdom
}

\begin{abstract}
Prefrontal cortex (PFC) is critical for self-ordered response sequencing. Patients with frontal lobe damage are impaired on response sequencing tasks, and increased blood flow has been reported in ventrolateral and dorsolateral PFC in subjects performing such tasks. Previously, we have shown that large excitotoxic lesions of the lateral PFC (LPFC) and orbitofrontal cortex FC (OFC), but not global prefrontal dopamine depletion, markedly impaired marmoset performance on a spatial self-ordered sequencing task (SSOST). To determine whether LPFC or OFC was responsible for the previously observed impairments and whether the underlying neural mechanism was modulated by serotonin, the present study compared the effects of selective LPFC and OFC excitotoxic lesions and 5,7-DHT-induced PFC serotonin depletions in marmosets on SSOST performance. Severe and long-lasting impairments in SSOST performance, including robust perseverative responding, followed LPFC but not OFC lesions. The deficit was ameliorated by task manipulations that precluded perseveration. Depletions of serotonin within LPFC and OFC had no effect, despite impairing performance on a visual discrimination reversal task, thus providing further evidence for differential monaminergic regulation of prefrontal function. In the light of the proposed attentional control functions of ventrolateral PFC and the failure of LPFC-lesioned animals to disengage from the immediately preceding response, it is proposed that this deficit may be due to a failure to attend to and register that a response has been made and thus should not be repeated. However, 5-HT does not appear to be implicated in this response inhibitory capacity.
\end{abstract}

\section{Introduction}

Patients with frontal lobe damage are impaired on a variety of self-ordered sequencing tasks (SOST) (Petrides and Milner 1982; Owen et al., 1990, 1995; Chase et al., 2008). Successful performance of such tasks requires varying degrees of working memory, monitoring of (attention to) actions, inhibitory control, and response planning, many of which are frequently associated with functioning of the lateral prefrontal cortex (LPFC) (Clark et al., 2007). Previously, using a spatial SOST (SSOST) in marmosets, we showed that large excitotoxic lesions of the PFC, which included lateral and orbital regions, as well as the anteroventral sector of the medial wall, impaired performance (Collins et al., 1998). In this version of the task, marmosets were presented with blocks of trials in which small squares (or boxes) were presented in two, three, or four (out of a possible eight) spatial locations on a touch-sensitive computer screen. On each trial, a different com-

Received Jan. 20, 2009; revised March 27, 2009; accepted March 27, 2009

This work was supported by a Wellcome Trust programme grant (to T.W.R., B.J. Everitt, A.C.R., and B.J. Sahakian) and conducted within the Medical Research Council Centre for Behavioural and Clinical Neuroscience. We thank David Theobald for histochemical assistance, Mercedes Arroyo for preparation of histological material, and Adrian Newman for graphical support.

Correspondence should be addressed to Dr. Angela C. Roberts, Department of Physiology, Development and Neuroscience, University of Cambridge, Downing Street, Cambridge CB2 3DY, UK. E-mail:acr4@cam.ac.uk. DOI:10.1523/JNEUROSCI.0312-09.2009

Copyright $\odot 2009$ Society for Neuroscience $\quad$ 0270-6474/09/296033-09\$15.00/0 bination of box locations was presented, and marmosets had to respond, once and once only, to each box in a self-determined sequence to receive food reward. The PFC lesion deficit was characterized by repetition of the immediately preceding response and was evident even in the performance of the easiest, two-box, problems. The impairment could, however, be reversed by preventing animals from repeating their immediately preceding response. This was achieved by removing the stimulus from the touch screen as soon as it had been chosen and not returning it until the animal had made the next response. This manipulation restored performance to presurgery levels in the three-box and four-box problems, suggesting that the deficit in response sequencing was due primarily to a failure to disengage from the previous response. In contrast, global PFC dopamine (DA) depletion was without effect, despite disrupting performance on a spatial delayed response task in the same study and having also been shown in a separate study to disrupt attentional selection (Crofts et al., 2001). Whether the prefrontal excitotoxic lesion deficit was due to damage in orbital FC (OFC) or LPFC regions could not be ascertained, damage to both regions having been associated with deficits in inhibitory control (Jones and Mishkin, 1972; Diamond and Goldman-Rakic, 1989; Dias et al., 1996; Wallis et al., 2001). Excitotoxic lesions of the PFC in nonhuman primates (Petrides 1991a,b, 1995) and neuroimaging studies in 
healthy human volunteers (Petrides et al., 1993; Owen et al., 1996) have implicated lateral regions of PFC in response sequencing, and so we hypothesized that area to be most likely responsible. However, a role for the OFC could not be ruled out given the perseverative responding seen in marmosets following OFC lesions (Dias et al., 1996; Wallis et al., 2001; Man et al., 2009).

The present study thus compared the effects of selective, excitotoxic lesions of the OFC and LPFC on SSOST performance. In addition, it also investigated the role of serotonergic modulation of response sequencing within PFC. We have previously shown that PFC serotonin depletion causes perseverative responding across a range of different tasks in marmosets (Clarke et al., 2004; Walker et al., 2006, 2008), and so the present study determined whether the perseverative deficits seen on the SSOST may similarly reflect a dysfunction of 5-HT-mediated behavioral control processes.

\section{Materials and Methods \\ Subjects}

Twelve experimentally naive common marmosets (Callithrix jacchus), 4 males and 8 females, housed in pairs. Five days per week they were fed $20 \mathrm{~g}$ of MP.E1 primate diet (Special Diet Services) plus two pieces of carrot and simultaneously had access to water for $2 \mathrm{~h}$. At the weekend, they received fruit, egg sandwiches, Marmoset Jelly, malt loaf, and Rusk and had ad libitum access to water. All procedures were performed in accordance with the United Kingdom Animals (Scientific Procedures) Act of 1986, under Project License 80/1770.

\section{Apparatus}

Behavioral testing took place in a specially designed automated test apparatus situated in a sound-attenuated chamber (Roberts et al., 1988). The marmoset is positioned in front of a touch-sensitive video display unit (VDU). Banana milkshake, which serves as a reward in the experiment, could be delivered through a licking spout located immediately in front of the VDU. Initially, marmosets were trained to respond to visual stimuli on the touch screen. A detailed account of preliminary training has been given previously (Roberts et al., 1994). Briefly, monkeys received the following sequence of training: familiarization with a milkshake reward, learning of a tone-reward contingency, and responding on the touch screen. In the first stage of training to respond on the touch screen, a large green rectangle $(3 \mathrm{~cm}$ high), which filled the width of the VDU, was presented in the center of the screen, with pieces of marshmallow stuck to the touch-sensitive area. This encouraged the monkeys to reach for the visible food reward, inevitably triggering the touch screen, leading to delivery of banana milkshake reward. Over days, the amount of marshmallow and size of the visual stimulus were reduced until monkeys were reliably and accurately making 30 responses or more to a square stimulus presented to the left and right of the center of the screen in $20 \mathrm{~min}$.

\section{Task 1: spatial self-ordered sequencing}

Preoperative training. Each monkey was trained to touch a blue square stimulus (box) presented in any one of eight possible locations on the touch screen. Correct responses were signaled by the disappearance of the stimuli and the onset of a tone that continued throughout a $5 \mathrm{~s}$ period, during which reinforcement was available. Delivery of reinforcement was contingent upon the monkey licking at the reinforcement spout. Each trial was preceded by a $3 \mathrm{~s}$ inter-trial interval. Once a monkey could readily respond to boxes in all eight possible locations, a second box was added; boxes in two-box trials were colored green. The first response to either box resulted in that square flashing yellow and the onset of the tone for $0.1 \mathrm{~s}$. Both boxes then disappeared from the screen for one-half of a second before reappearing in the same locations. A response to the same box again was incorrect and the trial was terminated, the screen went blank, and the house light was extinguished for $5 \mathrm{~s}$. A response to the other (previously untouched box) was correct and resulted in both boxes flashing yellow $(0.1 \mathrm{~s})$ and the onset of the tone, which continued throughout the $5 \mathrm{~s}$ reinforcement period. After a $3 \mathrm{~s}$ inter-trial interval,

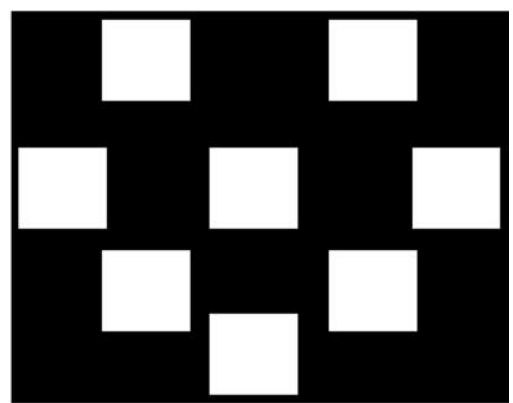

A) Box locations

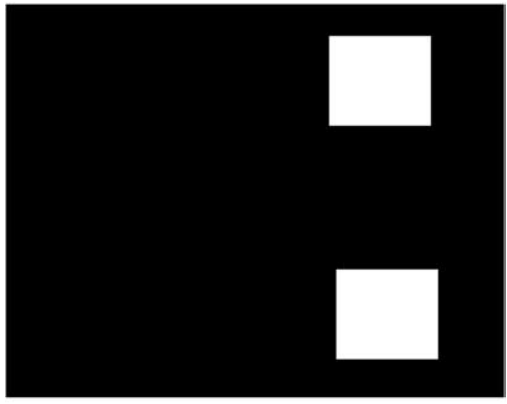

B) 2 Box problem

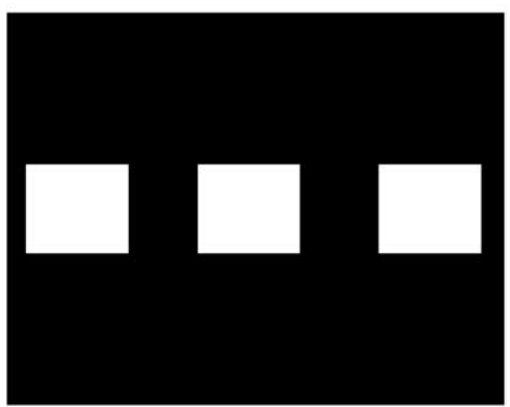

C) 3 Box problem

Figure 1. A shows the eight possible spatial locations at which a box could be presented on the touch screen. $\boldsymbol{B}$ and $\boldsymbol{C}$ show examples of a two-box and three-box problem. In each case, a monkey had to touch each of the boxes once and once only in a self-determined order to get reward.

the subsequent trial commenced with a novel array of two boxes. When performance improved, three-box trials were introduced. Boxes on three-box trials were colored blue; however, the task requirements remained the same- the monkey had to touch each box once, and once only in a self-determined order to obtain reward. Reinforcement was only available after successful completion of a given trial. If a monkey responded to any box in a given trial on more than one occasion, that trial was terminated and scored as incorrect. Each training session consisted of 15 two-box followed by 15 three-box problems. When monkeys were performing three-box problems at $>55 \%$ correct (chance performance, $33.3 \%$ correct), baseline performance was assessed.

Baseline performance. The baseline performance of each monkey was assessed across 10 sessions, each session comprising a standard set of 30 trials or problems, consisting of 15 trials of two-box problems and 15 trials of three-box problems (Fig. 1). A fixed set of trials was used each session, with order of trial presentation changing from day to day, although the 15 two-box problems always preceded 15 three-box problems. There then followed a single Probe session, which was a repeat of the fifth baseline session with the exception that a given trial did not 
Table 1. OFC, LPFC, and 5-HT lesion parameters, including the stereotaxic coordinates of each injection (based on the interaural plane) and injection volumes

\begin{tabular}{|c|c|c|c|c|c|c|c|c|c|c|c|c|}
\hline \multicolumn{4}{|l|}{ Orbital } & \multicolumn{4}{|l|}{ 5-HT } & \multicolumn{5}{|l|}{ Lateral } \\
\hline \multicolumn{3}{|c|}{ Coordinates (mm) } & \multirow{2}{*}{$\begin{array}{l}\text { Volume injected } \\
(\mu \mathrm{l})\end{array}$} & \multicolumn{3}{|c|}{ Coordinates (mm) } & \multirow{2}{*}{$\begin{array}{l}\text { Volume injected } \\
(\mu \mathrm{l})\end{array}$} & \multirow[b]{2}{*}{ Angle } & \multicolumn{3}{|c|}{ Coordinates (mm) } & \multirow{2}{*}{$\begin{array}{l}\text { Volume injected } \\
(\mu l)\end{array}$} \\
\hline AP & LM & V & & AP & LM & V & & & AP & LM & V & \\
\hline \multirow[t]{2}{*}{16.75} & \pm 2.5 & $0.7^{a}$ & 0.50 & 16.75 & \pm 1.0 & $0.7^{a}$ & 0.4 & $10^{\circ}$ & 16.0 & \pm 6.2 & $0.9^{a}$ & 1 \\
\hline & \pm 4.0 & $0.7^{a}$ & 0.40 & & \pm 2.5 & $0.7^{a}$ & 0.4 & $10^{\circ}$ & 16.0 & \pm 4.0 & $0.9^{a}$ & 1 \\
\hline \multirow[t]{2}{*}{17.75} & \pm 2.0 & $0.7^{a}$ & 0.50 & & \pm 4.0 & $0.7^{a}$ & 0.4 & $8^{\circ}$ & 16.75 & \pm 5.9 & $1.0^{a}$ & 1.6 \\
\hline & \pm 4.0 & $0.7^{a}$ & 0.40 & 17.75 & \pm 0.75 & $0.7^{a}$ & 0.4 & $8^{\circ}$ & 16.75 & \pm 5.9 & $1.5^{a}$ & 1.6 \\
\hline \multirow[t]{6}{*}{18.50} & \pm 2.0 & $0.7^{a}$ & 0.50 & & \pm 2.0 & $0.7^{a}$ & 0.4 & $8^{\circ}$ & 17.5 & \pm 5.6 & $1.0^{a}$ & 1.2 \\
\hline & \pm 4.0 & $0.7^{a}$ & 0.40 & & \pm 3.5 & $0.14^{b}$ & 0.4 & $8^{\circ}$ & 18.25 & \pm 5.3 & $1.0^{a}$ & 1.5 \\
\hline & & & & & \pm 4.5 & $0.14^{b}$ & 0.4 & $8^{\circ}$ & 19.0 & \pm 4.6 & $0.7^{a}$ & 0.8 \\
\hline & & & & 19.0 & \pm 0.75 & $0.7^{a}$ & 0.4 & Straight & 20 & \pm 3.0 & $1.7^{a}$ & 0.5 \\
\hline & & & & & \pm 2.0 & $0.7^{a}$ & 0.4 & & & & & \\
\hline & & & & & \pm 3.0 & $0.14^{b}$ & 0.4 & & & & & \\
\hline
\end{tabular}

OFC, LPFC, and 5-HT lesion parameters, including the stereotaxic coordinates of each injection (based on the interaural plane) and injection volumes.

${ }^{a}$ Above the base of the skull (for details, see Materials and Methods).

${ }^{b}$ Infusions of $0.4 \mu$ lat each of two depths, 0.7 and $1.4 \mathrm{~mm}$ above the base of the skull.

terminate after the first incorrect response. Instead, a monkey could make multiple errors with the maximum number of responses permitted, per trial, being twice the number of boxes on the screen +1 . Therefore, it was still possible to fail a given trial. In this Probe session, reinforcement was provided if all the boxes had been touched within the number of responses permitted.

Behavioral measures. The number of correct solutions was calculated separately for two-box and three-box problems within each of the 10 baseline sessions, providing a mean baseline score. In the Probe session, the number of perseverative errors, i.e., consecutive responses to the same box, was recorded.

\section{Surgical procedures}

Monkeys were premedicated with ketamine hydrochloride $(0.05 \mathrm{ml}$ of a $100 \mathrm{mg} / \mathrm{ml}$ solution i.m.; Pfizer), anesthetized with Saffan (aplphaxalone $0.9 \% \mathrm{w} / \mathrm{v}$ and alphadolone acetate $0.3 \% \mathrm{w} / \mathrm{v}, 0.4 \mathrm{ml}$ i.m.; ScheringPlough), and given a $24 \mathrm{~h}$ prophylactic analgesic (Rimadyl; $0.03 \mathrm{ml}$ of 50 $\mathrm{mg} / \mathrm{ml}$ carprofen s.c.; Pfizer) before being placed in a stereotaxic frame especially modified for the marmoset (David Kopf Instruments).

Excitotoxic lesions of the OFC. Neurons within the OFC were selectively destroyed by injecting $0.4-0.5 \mu \mathrm{l} /$ site of a $0.09 \mathrm{M}$ solution of quinolinic acid (in $0.1 \mathrm{~m}$ phosphate buffer, $\mathrm{pH} 7$; Sigma) into six sites, bilaterally within the PFC $(n=3)$. Each infusion was made through a stainless steel cannula (30 gauge) attached to a $2-\mu l$ syringe (Hamilton Bonaduz). See Table 1 for the stereotaxic coordinates.

Excitotoxic lesions of the LPFC. Neurons within the LPFC were selectively destroyed by injecting $0.5-1.6 \mu \mathrm{l} /$ site of a $0.09 \mathrm{M}$ solution of quinolinic acid (in $0.1 \mathrm{~m}$ phosphate buffer, $\mathrm{pH} 7$; Sigma) into eight sites, bilaterally within the PFC $(n=3)$. Each infusion was made through a stainless steel cannula (30 gauge) attached to a 10- $\mu$ l syringe (Hamilton Bonaduz). See Table 1 for the stereotaxic coordinates.

Serotonergic prefrontal cortex lesions. Selective serotonergic lesions of the PFC were made using 5,7-DHT (9.92 mm; Fluka BioChemika and Sigma) in saline $/ 0.1 \%$ L-ascorbic acid. To protect noradrenaline (NA) and DA innervations, the solution also contained the NA uptake blocker nisoxetine hydrochloride (50 mM; Sigma) and the DA uptake blocker GBR-12909 dihydrochloride (2.0 mm; Sigma).

Toxin solution was injected at a rate of $0.04 \mu \mathrm{l} / 20 \mathrm{~s}$ to 10 sites, bilaterally, using a 30 gauge cannula attached to a $2 \mu \mathrm{l}$ syringe (Hamilton Bonaduz). The stereotaxic coordinates are provided in Table 1.

\section{Postoperative testing}

After 1 week of postsurgical recovery, the performance of each monkey was assessed on (1) retention of baseline performance (10 sessions of 30 trials each, comprising fixed trial set 1 ) and a single probe session (Probe test 1), (2) a further series of 10 sessions comprising a novel set of 30 two-box and three-box trials, with boxes in novel combinations of spatial locations, followed by another probe session (Probe test 2), and (3) a modification of the basic paradigm in which perseveration (i.e., repetition of the immediately preceding response) was prevented. In this version of the task, when a box was touched, it disappeared from the touch screen and didn't reappear until an alternative box had been selected. Thus, it was still possible to fail trials with three squares, but it was impossible to do so by perseverating. Performance was measured over five sessions.

\section{Task 2: visual discrimination reversals}

Following completion of testing on the SSOST, 5-HT-lesioned and sham-lesioned control monkeys were tested on a set of visual discrimination reversals. We have previously shown that monkeys with 5-HT depletions of PFC are impaired in performance of this task (Clarke et al., $2004,2005)$ and thus was used here to confirm the functionality of the 5-HT depletion.

Behavioral protocol. Behavioral testing consisted of a series of twochoice discriminations composed of abstract colored stimuli. A response to the correct stimulus resulted in the incorrect stimulus disappearing from the screen, and onset of a $5 \mathrm{~s}$ tone that signaled the availability of $5 \mathrm{~s}$ of reinforcement. Following a response to the incorrect stimulus, both stimuli disappeared from the screen, and the house light was extinguished for $5 \mathrm{~s}$. The inter-trial interval was $3 \mathrm{~s}$, and within a session, the stimuli were presented equally to the left and right sides of the screen. Each monkey was presented with 30 trials per day, $5 \mathrm{~d}$ per week, and progressed to the next discrimination after attaining a criterion of $90 \%$ correct in the immediately preceding session. If a monkey showed a significant side bias (10 consecutive responses to one side), a rolling correction procedure (CP) was implemented whereby the correct stimulus was presented on the nonpreferred side until the monkey had made a total of three correct responses.

Monkeys first completed two simple visual discriminations. After acquisition of these discriminations to criterion of $90 \%$ correct in a single session, they acquired a third novel discrimination. Immediately following attainment of criterion performance on this discrimination, the stimulus reward contingencies were reversed, such that the previously unrewarded stimulus became rewarded, and the previously rewarded stimulus was unrewarded. Monkeys received two such reversals, having to reach criterion of $90 \%$ on the first before progressing onto the second.

Behavioral measure. The main measure of the monkeys' performance on the visual discriminations was the total number of errors made before achieving criterion of $>90 \%$ correct (excluding the day on which the criterion was attained) on each discrimination.

\section{Histological evaluation}

All marmosets that had received quinolinic acid lesions were deeply anesthetized with pentobarbitone and perfused transcardially with phosphate buffer $(0.1 \mathrm{~m}, \mathrm{pH} 7.3)$ followed by $4 \%$ paraformaldehyde, $\mathrm{pH}$ 7.3. Each brain was then removed and stored in fixative overnight before being 
transferred to $30 \%$ sucrose solution. A few days later, the tissue was sectioned at $60 \mu \mathrm{m}$, and every third section was mounted and stained with Cresyl Violet. A microscopic examination of the degree of neuronal cell loss and gliosis was then conducted, and the extent of each lesion was mapped onto standard drawings of coronal sections.

\section{Measurement of monoamines}

Postmortem tissue analysis using reversedphase HPLC assessed the specificity and extent of the selective 5,7-DHT lesions of the PFC. The exact methods used have been described in detail previously (Clarke et al., 2004, 2005). Briefly, 8-15 months after surgery, monkeys were killed, and their brains were removed and dissected on ice. Tissue samples were homogenized in $200 \mathrm{ml}$ of $0.2 \mathrm{M}$ perchloric acid and centrifuged at $6000 \mathrm{rpm}$ for $20 \mathrm{~min}$ at $4^{\circ} \mathrm{C}$. The supernatant was analyzed using reversed-phase HPLC and electrochemical detection. The signal was integrated using Chromeleon software (version 6.20; Dionex). The system was calibrated using standards containing known amounts of 5-HT, NA, and DA.

\section{Statistical methods}

Data were analyzed using SPSS version 16 . Repeated measures ANOVAs were used to analyze SOSST data and are described in detail in Results. Post hoc comparisons were made using appropriate post hoc tests (Cardinal and Aitken, 2006). Student's $t$ tests were used to analyze HPLC and serial discrimination reversal data. Where raw data did not display heterogeneity of variance, it was square root transformed (Howell, 1997).

\section{Results}

Histological analysis of excitotoxic lesions of LPFC and OFC

Schematic representations of the extent of the lesions of the LPFC and the OFC are illustrated in Figure 2. The intended lesion of the LPFC was the highly granular region lying on the lateral convexity from just behind the frontal pole to the beginning of the lateral ventricles, described by Burman et al. (2006), as similar in appearance to Walkers area 12/45. The resulting lesion encompassed the majority of this region in all three marmosets. It spared the very anterior sector unilaterally (section AP 18) in two marmosets and the most posterior sector (section AP 13) in one marmoset. In one or two of the marmosets, there was additional damage to the neighboring lateral regions of the OFC, more posteriorly (section AP 15) and to unilateral orbital and lateral regions in the frontal pole (sections AP 20 and 19).

The intended region of the OFC was the agranular and dysgranular regions lying on the orbitofrontal surface, anterior to the genu of the corpus callosum. The resulting lesions, in at least two of the three OFC-lesioned marmosets, included the majority of this area, sparing the ventromedial convexity and the neighboring highly granular regions of the lateral convexity. There was localized damage to parts of the medial wall, unilaterally (one animal, section AP 18; two animals, section AP 17).
A) Lateral prefrontal lesion

AP 20

AP 19

AP 18
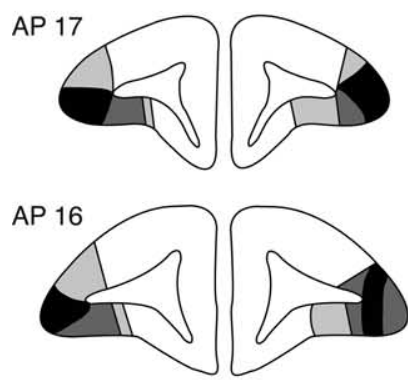

B) Orbitofrontal lesion

AP 20

AP 19

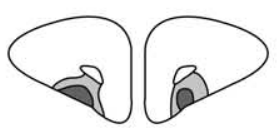

AP 18
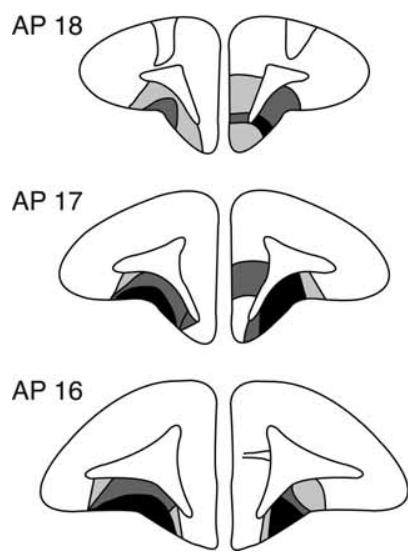
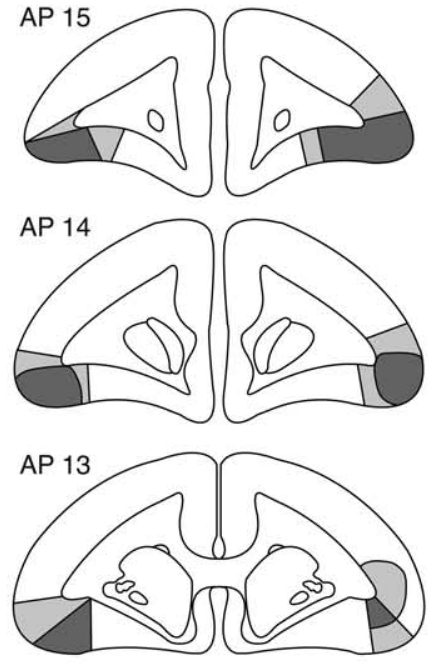

3 monkeys

2 monkeys

1 monkeys
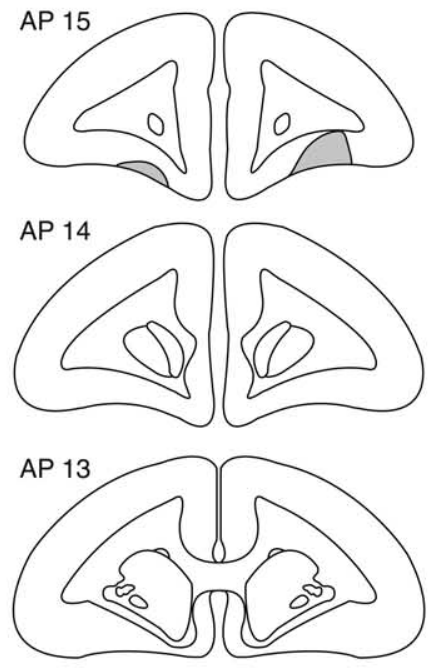
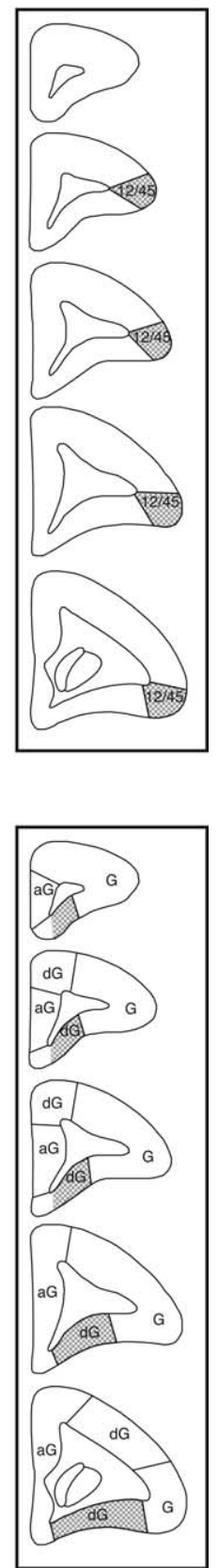

Figure 2. Schematic diagram of a series of coronal sections through the frontal lobe of the marmoset monkey, illustrating the extent of the LPFC $(\boldsymbol{A})$ and orbitofrontal $(\boldsymbol{B})$ lesions. The three decreasing shades of gray indicate regions that were lesioned in all three monkeys, any two monkeys, and one monkey, respectively. The inset coronal sections on the right indicate the region of $\operatorname{LPFC}(\boldsymbol{A})$ and OFC (B) that was targeted in this study.

\section{Postmortem serotonin depletions following 5,7-DHT} infusions into the PFC

Tissue analysis, 9-16 months postsurgery, revealed substantial depletions of 5-HT in both OFC and lateral regions of the PFC of 5,7-DHT-lesioned monkeys (Table 2). Analysis of postmortem tissue monoamine levels revealed significant reductions in 5-HT relative to sham-lesioned control subjects in the OFC $\left(t_{(4)}=3.72 ; p<\right.$ $0.05)$ and $\operatorname{LPFC}\left(t_{(4)}=5.25 ; p<0.01\right)$. There were no significant 5 -HT reductions in any other regions of the frontal lobe or subcortical regions (largest $t=2.61 ; p=0.06$ in B8). All other $t$ 's were $<1$. DA and NA levels were successfully protected, with no significant group differences in levels measured in any region (NA: largest $t=$ 
Table 2. Mean levels of 5-HT, dopamine, and noradrenaline (expressed as pmol/mg wet tissue weight \pm SEM) in the brain of control and lesion groups and the percentage depletion of serotonin ( \pm SEM) in marmosets with 5,7-DHT lesions of the frontal cortex ${ }^{a}$

\begin{tabular}{|c|c|c|c|c|c|c|c|}
\hline & \multicolumn{3}{|c|}{ 5-HT (pmol/mg) } & \multicolumn{2}{|c|}{ Dopamine (pmol/mg) } & \multicolumn{2}{|c|}{ Noradrenaline (pmol/mg) } \\
\hline & Control & Lesion & \% Depletion & Control & Lesion & Control & Lesion \\
\hline LPFC & $1.04(0.10)$ & $0.26(0.03)$ & $75.0(3.0)^{* *}$ & $0.29(0.03)$ & $0.27(0.04)$ & $1.11(0.04)$ & $0.91(0.2)$ \\
\hline $\mathrm{OFC}$ & $1.300 .18)$ & $0.45(0.09)$ & $65.3(6.9)^{*}$ & $0.43(0.08)$ & $0.66(0.24)$ & $1.29(0.13)$ & $0.87(0.17)$ \\
\hline Dorsal PFC & $0.70(0.07)$ & $0.45(0.05)$ & $34.7(6.5)$ & $0.59(0.14)$ & $0.43(0.10)$ & $1.44(0.14)$ & $1.02(0.09)$ \\
\hline Premotor/motor & $0.89(0.09)$ & $0.69(0.11)$ & $22.2(12.8)$ & $0.56(0.06)$ & $0.41(0.08)$ & $1.92(0.06)$ & $1.40(0.21)$ \\
\hline Medial PFC & $1.28(0.14)$ & $1.00(0.28)$ & $22.4(21.7)$ & $0.53(0.22)$ & $1.31(0.63)$ & $1.39(0.19)$ & $0.84(0.09)$ \\
\hline Anterior cingulate & $1.06(0.15)$ & $0.90(0.22)$ & $15.7(20.7)$ & $0.45(0.05)$ & $0.39(0.08)$ & $1.86(0.15)$ & $1.33(0.21)$ \\
\hline Mid-cingulate & $1.13(0.09)$ & $0.89(0.18)$ & $21.1(15.5)$ & $0.34(0.04)$ & $0.91(0.20)$ & $1.89(0.11)$ & $1.57(0.29)$ \\
\hline D.L. head of caudate & $1.95(0.43)$ & $1.60(0.48)$ & $17.7(19.6)$ & 84.4 (19.6) & $50.3(7.59)$ & $0.16(0.03)$ & $0.15(0.04)$ \\
\hline V.M. head of caudate & $2.04(0.37)$ & $2.00(0.28)$ & $1.90(13.5)$ & $88.8(14.48)$ & $68.2(2.77)$ & $0.29(0.07)$ & $0.48(0.21)$ \\
\hline Mid-caudate & $1.83(0.21)$ & $1.59(0.37)$ & $13.1(20.3)$ & $71.4(7.48)$ & $70.3(13.4)$ & $0.55(0.11)$ & $0.37(0.11)$ \\
\hline Anterior putamen & $2.25(0.37)$ & $1.58(0.30)$ & $29.6(13.7)$ & $75.5(13.22)$ & $44.4(5.04)$ & $0.32(0.05)$ & $0.88(0.17)$ \\
\hline Mid-putamen & $2.14(0.16)$ & $1.88(0.56)$ & $11.9(26.1)$ & $81.3(11.0)$ & $60.0(10.31)$ & $0.26(0.04)$ & $0.60(0.18)$ \\
\hline Nucleus accumbens & $3.31(0.71)$ & $2.73(0.88)$ & $17.6(26.6)$ & $54.9(11.95)$ & $35.0(5.35)$ & $3.52(1.78)$ & $2.81(0.99)$ \\
\hline Amygdala & $3.30(0.42)$ & $3.21(0.98)$ & $3.1(29.6)$ & $6.4(1.71)$ & $6.0(2.06)$ & $1.87(0.31)$ & $1.02(0.06)$ \\
\hline
\end{tabular}

${ }^{a}$ The asterisk indicates that mean scores of lesioned animals differ significantly from those of the control group when analyzed by independent samples $t$ test. OFC $\left(t_{4}=3.7 ;{ }^{*} p<0.02\right) ; \mathrm{LAT}$, lateral granular PFC $\left(t_{4}=5.25 ;{ }^{* *} p<0.006\right)$. In all cases, $n=3$ monkeys. D.L., Dorsolateral; V.M., ventromedical.

$2.196, p=0.093$ in medial PFC; $t=1.966, p=0.121$ in dorsal PFC; $t=1.609, p=0.183$ in premotor/motor; $t=1.361, p=0.25$ in $\mathrm{C} 1$; $t=1.35, p=0.25$ in OFC; all other $t^{\prime}$ s $<1$; DA: largest $t=1.59, p=$ 0.19 in anterior Putamen; all other $t^{\prime}$ 's, $t<1$ ).

\section{Behavioral assessment: SSOST}

\section{Baseline performance}

A two-way repeated measures ANOVA, with between-group factor of lesion and within group factor of difficulty (number of boxes), showed that there was no significant difference between lesion groups presurgery $(F<1)$, indicating that they were wellmatched for performance. There was a significant effect of difficulty $\left(F_{(1,3)}=144.5 ; p<0.0001\right)$, but no significant lesion by difficulty interaction $\left(F_{(3,8)}=3.3 ; p=0.08\right.$, NS). Thus, performance on two-box trials was significantly better than that on three-box trials.

\section{Accuracy}

Postsurgery, monkeys with excitotoxic lesions of the LPFC showed impaired performance on both two-box problems and three-box problems (Fig. 3). Neither of the other lesion groups (OFC excitotoxic; 5,7-DHT PFC) was affected. This is borne out by a repeated measures three-way ANOVA, with between group factor of lesion and within group factors of surgery (pre vs post) and difficulty (two boxes vs three boxes), which revealed a significant effect of surgery $\left(F_{(3,8)}=21.8 ; p<0.0001\right)$, significant effect of lesion $\left(F_{(1,3)}=95.2 ; p<0.0001\right)$, and a significant lesion $\times$ surgery interaction $\left(F_{(3,8)}=54.8 ; p<0.0001\right)$. There was also a significant effect of difficulty $\left(F_{(1,3)}=390.7 ; p<0.0001\right)$. However, there was no difficulty $X$ surgery or difficulty $\times$ lesion interaction. Analysis of simple main effects showed that performance of LPFC-lesioned monkeys was significantly worse after surgery, compared with baseline performance on both two-box $\left(F_{(1,4)}=32.643 ; p<0.005\right)$ and three-box $\left(F_{(1,4)}=94.35 ; p<\right.$ $0.001)$ trials. In contrast, the accuracy of the other groups did not differ between before surgery and after surgery.

\section{Probe test}

During the probe test session, trials did not terminate upon the first return to a previously selected box. In Figure 4 it can be seen that, compared with all other groups, LPFC-lesioned monkeys made many more perseverative errors (consecutive responses to the same box). A two-way repeated measures ANOVA, with between-subject factor of lesion and within subject factor of sur-

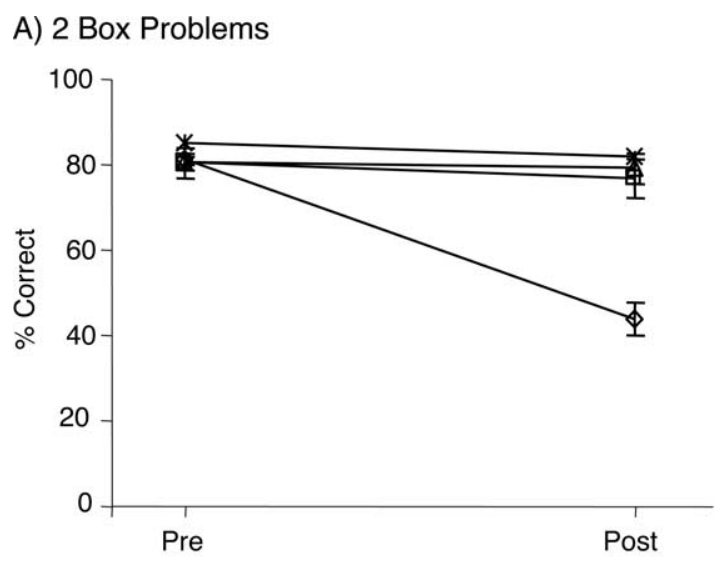

\section{B) 3 Box Problems}

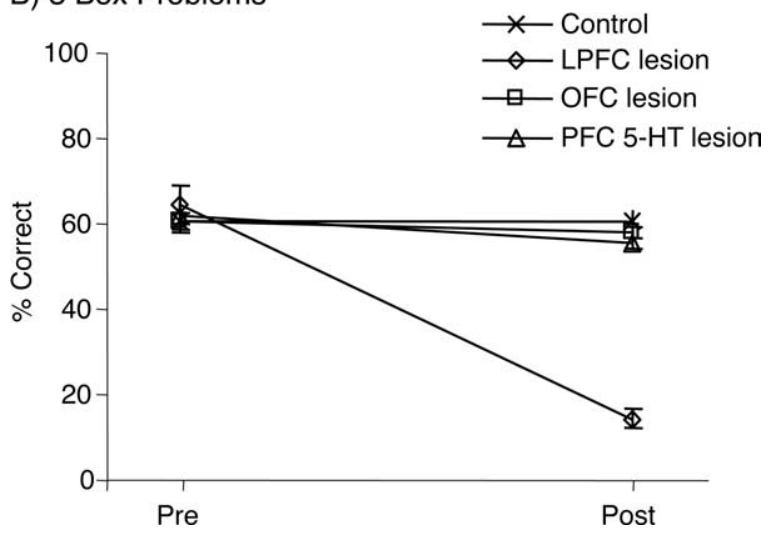

Figure 3. Percentage correct on two-box $(\boldsymbol{A})$ and three-box $(\boldsymbol{B})$ trials before surgery and after surgery. LPFC-lesioned monkeys made significantly more errors after surgery on both two-box and three-box trials compared with baseline performance $(p<0.01)$.

gery, revealed a significant effect of surgery $\left(F_{(3,8)}=36.6 ; p<\right.$ $0.0001)$, a significant effect of lesion $\left(F_{(1,3)}=6.312 ; p<0.04\right)$, and a significant lesion $\times$ surgery interaction $\left(F_{(3,8)}=9.1 ; p<\right.$ 0.006). Analysis of simple main effects revealed that LPFClesioned monkeys made significantly more perseverative errors on the postsurgery probe session than they did presurgery $\left(F_{(1,4)}\right.$ $=21.79 ; p<0.01)$. The performance of the other groups postsurgery did not differ from presurgery baseline. 
A) Probe Test: Pre-Surgery

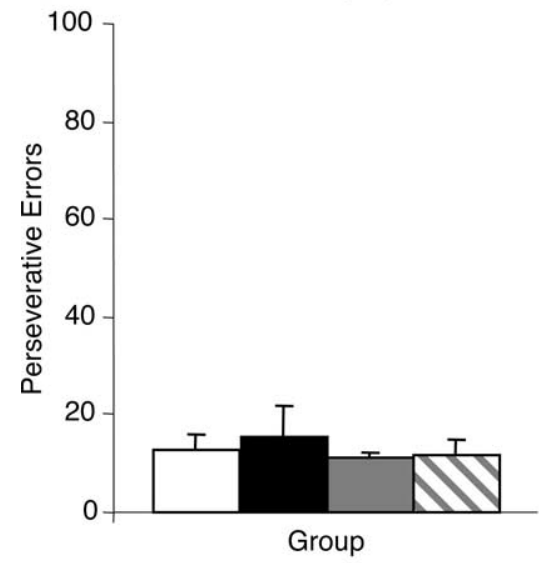

B) Probe Test: Post-Surgery

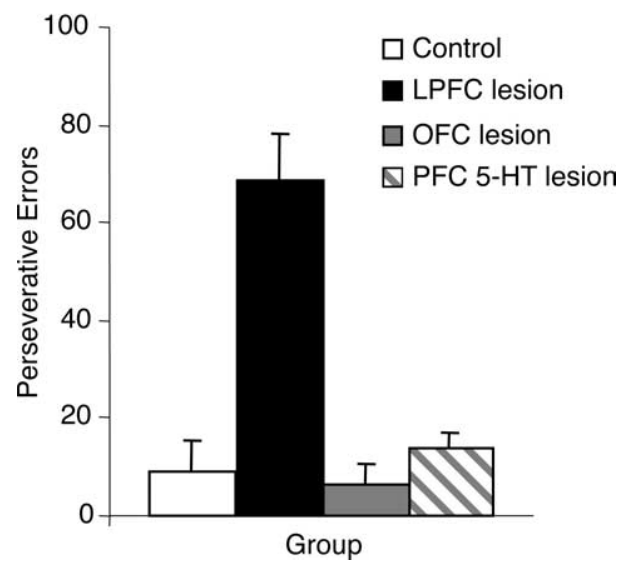

Figure 4. Number of perseverative errors (consecutive responses to the same box) made on the probe test before surgery $(\boldsymbol{A})$ and after surgery $(\boldsymbol{B})$. LPFC-lesioned monkeys made significantly more perseverative errors after surgery than they did before surgery $(p<0.01)$.

\section{Novel trial set}

The high levels of performance on the novel trial set confirmed the proficiency of the control, OFC-lesioned, and 5,7-DHTlesioned groups on the task. The lateral lesioned monkeys however, showed continued impairment (Fig. 5A). Repeatedmeasures two-way ANOVA, with between-group factor of lesion and within-group factor of difficulty, revealed a significant effect of lesion $\left(F_{(3,8)}=7.9 ; p=0.009\right)$ and difficulty $\left(F_{(1,3)}=87.2 ; p<\right.$ $0.0001)$, but no lesion $\times$ difficulty interaction $\left(F_{(3,8)}=1.89 ; p=\right.$ 0.2 ). Post hoc analysis using the Sidak test showed lateral lesioned monkeys performance on the novel trial set to be significantly worse than that of any of the other groups $(p<0.05)$.

On the novel probe session, again, lateral-lesioned monkeys made more perseverative errors than any of the other groups (Fig. $5 B$ ). Analysis of the square route transformed data using a oneway ANOVA showed there was significant difference between groups $\left(F_{(3,8)}=5.97 ; p=0.02\right)$. Post hoc analysis using Dunnett's $t$ test showed that only lateral-lesioned monkeys made significantly more errors than controls $(p<0.02)$.

\section{Self-ordered sequencing in the absence of perseveration} (perseveration test)

The perseverative nature of the lateral-lesioned monkeys deficit is further demonstrated by their improved performance on a modified version of the task in which they were prevented from reselecting the previously selected box. On this version, accuracy on
A) Novel Trial Set: Accuracy

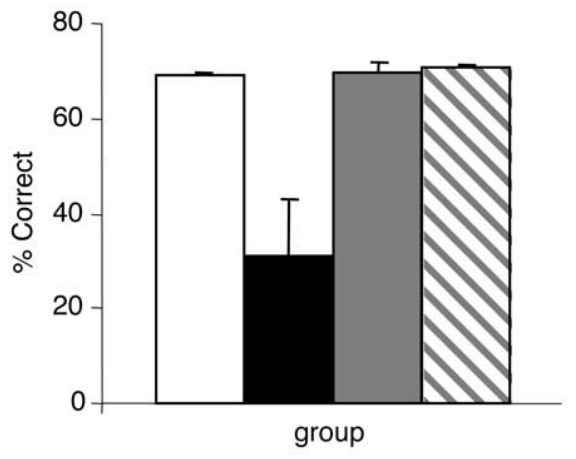

B) Novel Trial Set: Probe Test

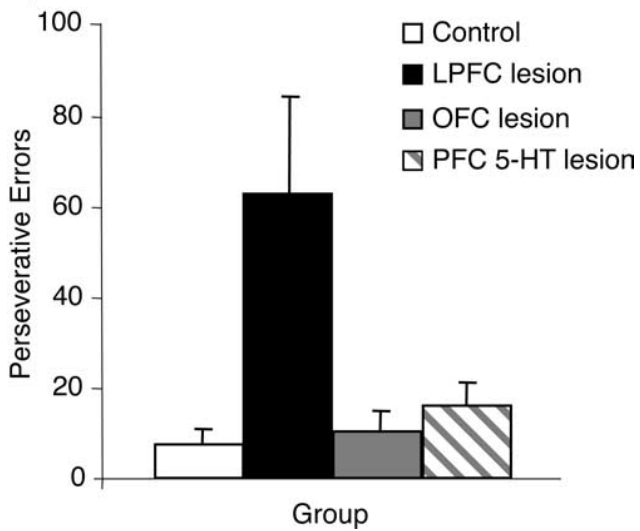

Figure 5. A, Overall accuracy on the novel trial set. 5 -HT-lesioned and $0 \mathrm{FC}-$ lesioned monkeys continued to show high levels of performance. LPFC-lesioned monkeys performance was significantly worse than any of the other groups $(p<0.05)$. $\boldsymbol{B}, 0$ n the novel probe session, lateral-lesioned monkeys made significantly more perseverative errors than controls $(p<0.02)$.

Performance of LPFC lesioned animals

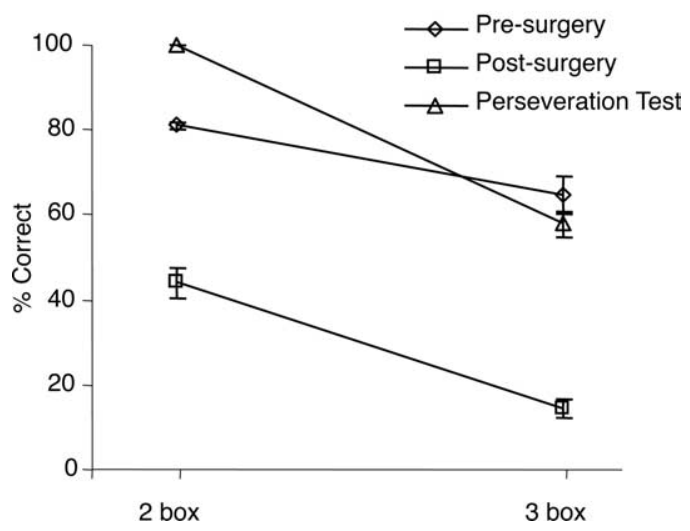

Figure 6. Performance of the LPFC-lesioned monkeys on a modified version of the task, which prevented perseverative responding in comparison with presurgery and postsurgery two-box and three-box accuracy. When prevented from reselecting the previously selected box, accuracy on three-box trials returned to presurgery levels ( $p=0.2$, NS) and was significantly better than postsurgery performance $(p<0.0001)$.

three-box trials returned to presurgery levels (Fig. 6). This was confirmed by statistical analysis using a two-way ANOVA comparing control and lateral-lesioned monkeys performance before surgery, after surgery, and on the modified version of the task. There was a significant effect of group $\left(F_{(1,4)}=38.6 ; p<0.003\right)$, 
Table 3. Errors to criterion for individual monkeys from the control and 5,7-DHTlesioned groups for each of three visual discriminations (D1-D3)

\begin{tabular}{|c|c|c|c|}
\hline & D1 & D2 & D3 \\
\hline \multicolumn{4}{|l|}{ Control } \\
\hline M1 & 25 & 41 & 49 \\
\hline M2 & 62 & 136 & 43 \\
\hline Mean \pm SEM & $56.5 \pm 5.5$ & $88.5 \pm 47.4$ & $46 \pm 3.0$ \\
\hline \multicolumn{4}{|l|}{ 5,7-DHT lesion } \\
\hline M1 & 25 & 108 & 35 \\
\hline M2 & 118 & 101 & 24 \\
\hline M3 & 273 & 300 & 213 \\
\hline Mean \pm SEM & $138.7 \pm 72.3$ & $169.7 \pm 65.2$ & $87.3 \pm 63.1$ \\
\hline
\end{tabular}

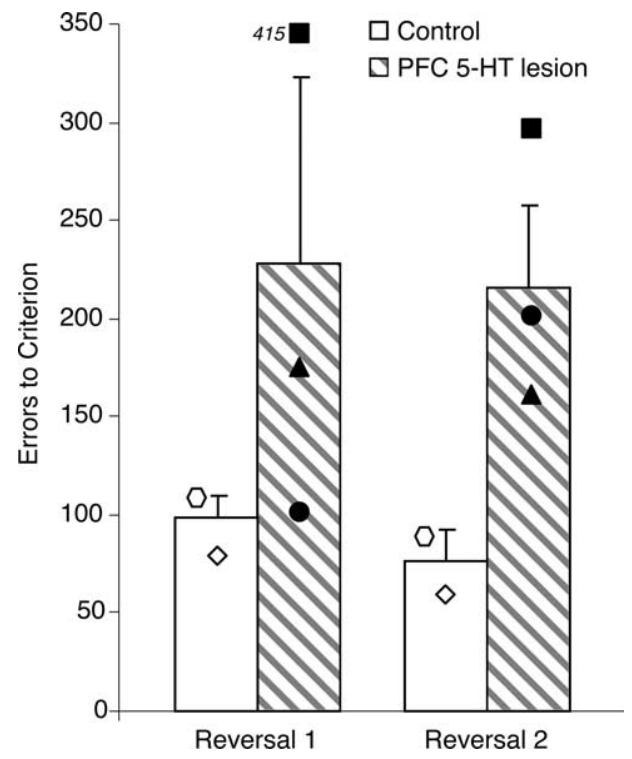

Figure 7. Total errors (excluding $(P$ trials) made before attaining criterion performance on the serial discrimination reversals (R1, R2). Individual animal error scores are represented by symbols, and bars represent SEM. The $95 \%$ confidence intervals of the 5 -HT-lesioned group did not overlap those of the control group for reversal 2, and thus, the groups were significantly different from one another. Only one control and one 5-HT-lesioned animal received any CP trials (control R1:10, R2:14; lesion R1:41, R2:13).

a significant effect of trial set (before surgery, after surgery, and modified; $\left.F_{(1,4)}=233.1 ; p<0.0001\right)$, and a significant group $\times$ trial set interaction $\left(F_{(1,4)}=201.4 ; p<0.001\right)$. Analysis of simple main effects revealed that, as expected, performance of control monkeys did not differ across trial sets $\left(F_{(2,6)}=0.99 ; p=0.4\right.$, NS). However, performance of lateral-lesioned monkeys did $\left(F_{(2,6)}=61.8 ; p<0.0001\right)$. Post hoc analysis using Fisher's LSD showed that when lateral-lesioned monkeys were prevented from perseverating, their accuracy did not differ from presurgery levels $(p=0.228$, NS) and was significantly better than postsurgery performance $(p<0.0001)$. The fact that the same modification had no effect on the accuracy of control monkeys suggests that the benefit seen in the lateral-lesioned group is not a consequence of reduced task demands, in general.

\section{Behavioral assessment: visual discrimination reversal}

Unfortunately, one of the control monkeys had to be killed before it could be tested on the serial visual discrimination reversal task. Thus, the three prefrontal 5-HT-lesioned animals could only be compared with two sham-operated controls. There was no difference between the groups in acquiring a series of three visual discriminations. Two of the three prefrontal 5-HT-lesioned ani- mals performed equivalently to the two controls (Table 3). Only one of the lesioned animals made many more errors, but this lesioned animal did not differ with respect to the percentage loss of 5-HT, or the other monoamines, within the PFC or related regions, to that of the other two. In contrast to acquisition, it can be seen in Figure 7 that all three 5-HT-lesioned monkeys made more errors to reach criterion following reversal of the stimulus reward contingencies than the two controls, especially on reversal 2. Despite the low sample sizes, the $95 \%$ confidence intervals for the mean of the square root transformed total errors of the two groups were nonoverlapping for reversal 2 but not for reversal 1 (reversal 1: control, mean $=9.85$, confidence limit $=1.08$; lesion, mean 14.4 , confidence limit $=6.07$; reversal 2 : control, mean $=$ 7.15 , confidence limit $=1.27$; lesion, mean 14.7 , confidence limit $=3.12$ ). This finding is entirely consistent with three previous studies showing that depletion of prefrontal 5-HT causes discrimination reversal deficits (Clarke et al., 2004, 2005, 2007).

\section{Discussion}

The present investigation demonstrates that excitotoxic lesions of the LPFC, but not the OFC, in marmosets causes severe and long-lasting impairments in SSOST performance. However, 5,7DHT-induced serotonin depletions throughout LPFC and OFC had no effect of SSOST performance, despite impairing acquisition of a visual discrimination reversal task.

The SSOST requires animals to respond, in a self-determined sequence, once, and once only, to each of either two or three spatial locations on a touch-sensitive computer screen, to receive liquid reward (two-box and three-box problems). Before surgery, all animals were responding significantly above chance, but after surgery, the performance of LPFC-lesioned marmosets declined to chance levels. Impaired performance was seen across the entire postoperative 10 -session retention test and persisted across a further 10 sessions involving novel two-box and three-box problems. In the standard task, an error resulted in the termination of the trial, but to gain further insight into the nature of the impairment, the animals performed a probe test in which the first error did not result in trial termination but instead, animals could continue to respond until all locations had been selected, at which point reward was received (probe test). Under these conditions, LPFC-lesioned animals displayed profound perseverative responding, repeating the immediately preceding response over and over again. In contrast, lesioned animals from all other groups made few such errors.

This failure to avoid the reselection of the previously chosen location was shown to underlie their general impairment on the SSOST. Thus, the performance of LPFC-lesioned animals returned to preoperative levels, if, after a spatial location had been selected, the box representing that spatial location was removed from the screen and only returned after the animal had made another response (perseveration test). In the two-box problems, this manipulation removed any working memory requirement because, after their first selection, there was only one other box remaining on the screen. However, in the three-box problems, the third response still required the animal to choose between two spatial locations, one of which had not been selected on that trial and one of which that had already been selected, and thus should have been avoided. It may be argued that this manipulation converts the three-box problem into a far easier, two-box problem, but it should be noted that, within the standard task, LPFClesioned animals performed two-box problems equally as poorly as three-box problems. If the performance of LPFC-lesioned animals on three-box problems, when repeating the immediately 
preceding response was prevented (Fig. $6, \triangle$ ), is compared with their performance on standard two-box problems (Fig. $3, \diamond$ ), it can be seen that the former is still superior, despite these threebox problems ostensibly acting as two-box problems. This further emphasizes the fact that the failure to avoid the reselection of the previously chosen spatial location underlies the deficit in these animals.

That LPFC, but not OFC, lesions disrupted response sequencing in marmosets is consistent with the finding of an increase in the BOLD response within the lateral PFC of humans when performing a similar spatial, response sequencing task (Owen et al., 1996). In this latter study, blood flow changes occurred in either the ventrolateral and/or dorsolateral regions of PFC, depending on the precise cognitive and executive demands. Specifically, the organization and execution of a sequence of spatial moves produced significant activations in the ventrolateral PFC (area 47). Although there is evidence for elements of both dorsolateral and ventrolateral granular regions within marmoset PFC, whether they map onto areas 46, 9, and 47/12 (Petrides and Pandya, 1999, 2002) of humans and rhesus monkeys remains unclear. However, based on the cytoarchitectonic map of marmoset PFC (Burman et al., 2006), the LPFC lesion in the present study included area $45 / 47$, but left intact, areas 46 and 9 . Thus, the present study demonstrates that lesions of a region in marmosets, cytoarchitectonically similar to area 45/47 in humans, profoundly disrupts response sequencing, extending a previous imaging study demonstrating an increase in blood flow within area 47 during response sequencing.

The precise role of ventrolateral PFC remains to be determined. It has been implicated in inhibitory control functions because of its involvement in tests that require inhibition, such as attentional set-shifting, go-no go and a stop signal reaction time tasks (Dias et al., 1996; Rubia et al., 2001; Aron et al., 2003, 2004; Clark et al., 2007). However, any contribution made by ventrolateral PFC to inhibitory control is distinct from that of the OFC. For example, we have previously shown that both LPFC and OFC lesions produce dissociable deficits in performance of an object retrieval task. Monkeys with OFC, but not LPFC, lesions are impaired at learning to make a detour reach around a transparent barrier to retrieve reward, failing to inhibit their prepotent response tendency to reach directly along their line of site to the visible reward. In contrast, if the detour reach has been learned previously in the context of an opaque barrier, LPFC-lesioned, but not OFC-lesioned, animals are impaired on transferring that response to the transparent version, displaying instead direct line of sight reaching (Wallis et al., 2001). In the SSOST, there is no direct link between a particular stimulus/response and reward, which has to be inhibited, as in the standard version of the object retrieval task. Instead, tracking a series of responses rather than learning about stimulus reward contingencies is required for successful performance. Thus, it might be the tracking of actions as opposed to contingencies that makes the task laterally, as opposed to orbitally, mediated.

Recently, this LPFC region has been implicated in attentional control mechanisms (Hampshire et al., 2007), as distinct from inhibitory control mechanisms. The region is commonly activated in tasks without a requirement for inhibitory control but with a strong attentional component such as target detection (Linden et al., 1999; Hampshire et al., 2007, 2008) and attentional reorienting (Corbetta and Shulman, 2002). Moreover, neuronal activity in this region rapidly adapts to code for task-relevant inputs in a variety of different contexts (Freedman et al., 1998; Duncan 2001; Miller and Cohen, 2001). Thus, an impaired ability to disengage from the immediately preceding response seen in LPFC-lesioned marmosets on the SSOST may be the result of a failure to attend to and register that a response was made and thus should not be repeated. Future studies should determine whether enhancing the saliency of response feedback reduces the deficit. There are several clinical implications of this work; for example, Clark et al. (2007) have shown that self-ordered spatial working memory performance in adult attention deficit/hyperactivity disorder can be linked to putative malfunctions of this LPFC region. Furthermore, the perseverative responding of the LPFC-lesioned marmosets was reminiscent of the compulsive "checking" of obsessive-compulsive disorder patients (Rasmussen and Eisen 1991), which prevents them moving to the next behavioral sequence (Chamberlain et al., 2006).

As well as determining the region of PFC critical for selfordered response sequencing, this study also addressed the question of whether the underlying behavioral control mechanism was modulated by serotonin. Previously, we have reported deficits in a range of inhibitory control tasks following widespread depletion of serotonin within the marmoset PFC (Clarke et al., 2004; Walker et al., 2006, 2009). In the majority of cases, the serotonin-depleted animals displayed perseverative responding to a previously rewarded stimulus, although we also show that perseverative responding can occur to visually salient stimuli that have no history of reward (Walker et al., 2009). Consistent with these findings, animals with serotonin depletions within LPFC and OFC in the present study displayed perseverative responding on a discrimination reversal task but, in contrast, exhibited intact performance on the SSOST. The depletions were of a similar magnitude across both the OFC and LPFC and were sufficient to cause impairment in orbitofrontal-dependent reversal learning. Thus, it is unlikely that the failure to show a deficit in the SSOST task was due to the LPFC, 5-HT depletion being of insufficient magnitude. A more likely explanation is that the underlying mechanisms responsible for response sequencing are not dependent on serotonin modulation. They are also independent of DA modulation as 6-OHDA-induced depletions of prefrontal catecholamines, which disrupt the ability of marmosets to develop an attentional set (Crofts et al., 2001), did not disrupt SSOST performance (Collins et al., 1998). It is plausible that PFC NA, which was incompletely depleted in the latter study, has a role, especially as PFC NA has been implicated in attentional setshifting in rodents (Lapiz and Morilak, 2006; Tait et al., 2007; Newman et al., 2008) and in the modulation of the human LPFC (Chamberlain et al., 2008). Whatever this putative role of PFC $\mathrm{NA}$, the present findings for PFC serotonin are relevant to the growing body of evidence for differential regulation of frontoexecutive functioning by the monoamines, according to the PFC region engaged (Robbins and Roberts, 2007).

\section{References}

Aron A, Fletcher P, Bullmore E, Sahakian B, Robbins T (2003) Stop-signal inhibition disrupted by damage to right inferior frontal gyrus in humans. Nat Neurosci 6:115-116.

Aron AR, Monsell S, Sahakian BJ, Robbins TW (2004) A componential analysis of task-switching deficits associated with lesions of left and right frontal. Brain 127:1561-1573.

Burman K, Palmer S, Gamberini M, Rosa M (2006) Cytoarchitectonic subdivisions of the dorsolateral frontal cortex of the marmoset monkey ( Callithrix jacchus), and their projections to dorsal visual areas. J Comp Neurol 495:149-172.

Cardinal RN, Aitken MRF (2006) ANOVA for the behavioural sciences researcher. Mahwah, NJ: Laurence Erlbaum Associates.

Chamberlain SR, Blackwell AD, Fineberg NA, Robbins TW, Sahakian BJ 
(2006) Strategy implementation in obsessive-compulsive disorder and trichotillomania. Psychol Med 36:91-97.

Chamberlain SR, Hampshire A, Müller U, Rubia K, Campo ND, Craig K, Regenthal R, Suckling J, Roiser JP, Grant JE, Bullmore ET, Robbins TW, Sahakian BJ (2008) Atomoxetine modulates right inferior frontal activation during inhibitory control: a pharmacological functional magnetic resonance imaging study. Biol Psychiatry 65:550-555.

Chase HW, Clark L, Sahakian BJ, Bullmore ET, Robbins TW (2008) Dissociable roles of prefrontal subregions in self-ordered working memory performance. Neuropsychologia 46:2650-2661.

Clark L, Blackwell AD, Aron AR, Turner DC, Dowson J, Robbins TW, Sahakian BJ (2007) Association between response inhibition and working memory in adult ADHD: a link to right frontal cortex pathology? Biol Psychiatry 61:1395-1401.

Clarke H, Walker S, Dalley J, Robbins T, Roberts A (2007) Cognitive inflexibility after prefrontal serotonin depletion is behaviorally and neurochemically specific. Cereb Cortex 17:18-27.

Clarke HF, Dalley JW, Crofts HS, Robbins TW, Roberts AC (2004) Cognitive inflexibility after prefrontal serotonin depletion. Science 304:878-880.

Clarke HF, Walker SC, Crofts HS, Dalley JW, Robbins TW, Roberts AC (2005) Prefrontal serotonin depletion affects reversal learning but not attentional set shifting. J Neurosci 25:532-538.

Collins P, Roberts AC, Dias R, Everitt BJ, Robbins TW (1998) Perseveration and strategy in a novel spatial self-ordered sequencing task for nonhuman primates: effects of excitotoxic lesions and dopamine depletions of the prefrontal cortex. J Cogn Neurosci 10:332-354.

Corbetta M, Shulman GL (2002) Control of goal-directed and stimulusdriven attention in the brain. Nat Rev Neurosci 3:201-215.

Crofts HS, Dalley JW, Collins P, Van Denderen JCM, Everitt BJ, Robbins TW, Roberts AC (2001) Differential effects of 6-OHDA lesions of the frontal cortex and caudate nucleus on the ability to acquire an attentional set. Cereb Cortex 11:1015-1026.

Diamond A, Goldman-Rakic PS (1989) Comparison of human infants and rhesus monkeys on Piaget's AB task: evidence for dependence on dorsolateral prefrontal cortex. Exp Brain Res 74:24-40.

Dias R, Robbins TW, Roberts AC (1996) Dissociation in prefrontal cortex of affective and attentional shifts. Nature 380:69-72.

Duncan J (2001) An adaptive coding model of neural function in prefrontal cortex. Nat Rev Neurosci 2:820-829.

Freedman M, Black S, Ebert P, Binns M (1998) Orbitofrontal function, object alternation and perseveration. Cereb Cortex 8:18-27.

Hampshire A, Duncan J, Owen AM (2007) Selective tuning of the blood oxygenation level-dependent response during simple target detection dissociates human frontoparietal subregions. J Neurosci 27:6219-6223.

Hampshire A, Gruszka A, Fallon SJ, Owen AM (2008) Inefficiency in selforganized attentional switching in the normal aging population is associated with decreased activity in the ventrolateral prefrontal cortex. J Cogn Neurosci 20:1670-1686.

Howell DC (1997) Statistical methods for psychology, Ed 5. Belmont, CA: Wadsworth Publishing.

Jones B, Mishkin M (1972) Limbic lesions and the problem of stimulusreinforcement associations. Exp Neurol 36:362-377.

Lapiz MD, Morilak DA (2006) Noradrenergic modulation of cognitive function in rat medial prefrontal cortex as measured by attentional set shifting capability. Neuroscience 137:1039-1049.

Linden D, Prvulovic D, Formisano E, Völlinger M, Zanella F, Goebel R, Dierks T (1999) The functional neuroanatomy of target detection: an fMRI study of visual and auditory oddball tasks. Cereb Cortex 9:815-823.

Man MS, Clarke HF, Roberts AC (2009) The role of the orbitofrontal cortex and medial striatum in the regulation of prepotent responses to food rewards. Cereb Cortex 19:899-906.

Miller EK, Cohen JD (2001) An integrative theory of prefrontal cortex function. Annu Rev Neurosci 24:167-202.
Newman LA, Darling J, McGaughy J (2008) Atomoxetine reverses attentional deficits produced by noradrenergic deafferentation of medial prefrontal cortex. Psychopharmacology 200:39-50.

Owen A, Doyon J, Petrides M, Evans A (1996) Planning and spatial working memory: a positron emission tomography study in humans. Eur J Neurosci 8:353-364.

Owen AM, Downes JJ, Sahakian BJ, Polkey CE, Robbins TW (1990) Planning and spatial working memory following frontal lobe lesions in man. Neuropsychologia 28:1021-1034.

Owen AM, Sahakian BJ, Semple J, Polkey CE, Robbins TW (1995) Visuospatial short-term recognition memory and learning after temporal lobe excisions, frontal lobe excisions or amygdalo-hippocampectomy in man. Neuropsychologia 33:1-24

Petrides M (1991a) Functional specialization within the dorsolateral frontal cortex for serial order memory. Proc Biol Sci 246:299-306.

Petrides M (1991b) Monitoring of selections of visual stimuli and the primate frontal cortex. Proc Biol Sci 246:293-298.

Petrides M (1995) Impairments on nonspatial self-ordered and externally ordered working memory tasks after lesions of the mid-dorsal part of the lateral frontal cortex in the monkey. J Neurosci 15:359-375.

Petrides M, Milner B (1982) Deficits on subject-ordered tasks after frontaland temporal-lobe lesions in man. Neuropsychologia 20:249-262.

Petrides M, Pandya D (1999) Dorsolateral prefrontal cortex: comparative cytoarchitectonic analysis in the human and the macaque brain and corticocortical connection patterns. Eur J Neurosci 11:1011-1036.

Petrides M, Pandya D (2002) Comparative cytoarchitectonic analysis of the human and the macaque ventrolateral prefrontal cortex and corticocortical connection patterns in the monkey. Eur J Neurosci 16:291-310.

Petrides M, Alivisatos B, Evans A, Meyer E (1993) Dissociation of human mid-dorsolateral from posterior dorsolateral frontal cortex in memory processing. Proc Natl Acad Sci U S A 90:873-877.

Rasmussen S, Eisen JL (1991) Phenomenology of OCD: clinical subtypes, heterogeneity and coexistence. In: The psychology of obsessive-compulsive disorder (Zohar J, Insel T, Rasmussen S, eds), pp 13-43. New York: Springer.

Robbins TW, Roberts AC (2007) Differential regulation of fronto-executive function by the monoamines and acetylcholine. Cereb Cortex 17:i151-i160.

Roberts A, De Salvia M, Wilkinson L, Collins P, Muir J, Everitt B, Robbins T (1994) 6-Hydroxydopamine lesions of the prefrontal cortex in monkeys enhance performance on an analog of the Wisconsin Card Sort Test: possible interactions with subcortical dopamine. J Neurosci 14:2531-2544.

Roberts AC, Robbins TW, Everitt BJ (1988) The effects of intradimensional and extradimensional shifts on visual discrimination learning in humans and nonhuman primates. Q J Exp Psychol B 40:321-341.

Rubia K, Russell T, Overmeyer S, Brammer MJ, Bullmore ET, Sharma T, Simmons A, Williams SCR, Giampietro V, Andrew CM, Taylor E (2001) Mapping motor inhibition: conjunctive brain activations across different versions of go/no-go and stop tasks. Neuroimage 13:250-261.

Tait DS, Brown VJ, Farovik A, Theobald DE, Dalley JW, Robbins TW (2007) Lesions of the dorsal noradrenergic bundle impair attentional set-shifting in the rat. Eur J Neurosci 12:3719-3724.

Walker SC, Mikheenko YP, Argyle LD, Robbins TW, Roberts AC (2006) Selective prefrontal serotonin depletion impairs acquisition of a detourreaching task. Eur J Neurosci 23:3119-3123.

Walker SC, Robbins TW, Roberts AC (2009) Differential contributions of dopamine and serotonin to orbitofrontal cortex function in the marmoset. Cereb Cortex 19:889-898.

Wallis JD, Dias R, Robbins TW, Roberts AC (2001) Dissociable contributions of the orbitofrontal and lateral prefrontal cortex of the marmoset to performance on a detour reaching task. Eur J Neurosci 13:1797-1808. 